\title{
Two-Phase Flow Pressure Drop in Superhydrophobic Channels
}

\author{
Kimberly A. Stevens, Julie Crockett, Daniel R. Maynes, Brian D. Iverson \\ Department of Mechanical Engineering \\ Brigham Young University \\ Provo, UT USA
}

\begin{abstract}
Superhydrophobic surfaces promote dropwise condensation, which increases the rate of thermal transport, making them desirable for use in condensers. Adiabatic two-phase flow loops have been constructed to gain insight into the hydrodynamics of two-phase systems, laying the groundwork for further study of condensing flow on superhydrophobic surfaces. A two-phase flow loop to measure pressure drop and visualize the flow patterns of two-phase flow in superhydrophobic channels relative to classical hydrophilic and hydrophobic surfaces was built and validated. Good agreement was found between observed pressure drops and theoretical and experimental correlations in the literature.
\end{abstract}

\section{Introduction}

Superhydrophobic surfaces have recently received much media attention because of their excellent water-repelling capabilities. Commonly, they have micro- or nano-scale surface features combined with a hydrophobic coating, resulting in high contact angles (greater than $\left.150^{\circ}\right)$. An example of two different superhydrophobic surfaces is shown in Figure 1. Superhydrophobic surfaces have been shown to promote drop-wise condensation which increases the rate of thermal transport during condensation by an order of magnitude, relative to film-wise condensation [1-5]. In addition, superhydrophobic surfaces can significantly decrease the pressure drop in channel flow. The combination of these benefits makes superhydrophobic surfaces very attractive for use in flow-condensing environments (e.g., in thermal management of electronics, desalination, loop heat pipes, and power generation).

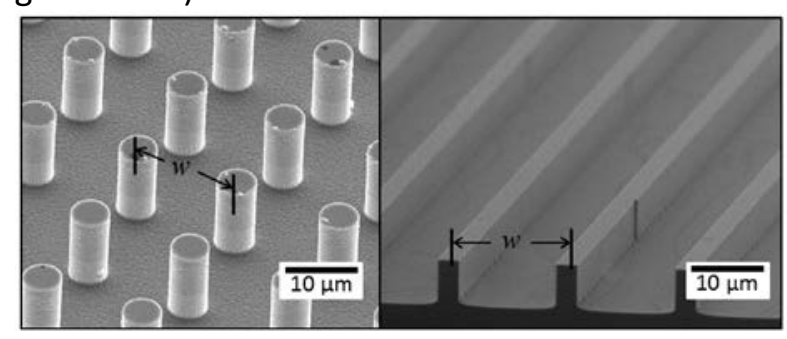

Figure 1--SEM image of a superhydrophobic surface

Despite the potential applications, little is known about condensation behavior on superhydrophobic surfaces in the presence of vapor flow. Shear forces from the vapor flow are particularly important in zero-gravity environments to remove the droplets from the surface for additional condensate to form, but thus far only one paper regarding flowcondensation on superhydrophobic surfaces has been published [6]. Further research is needed to fully understand the behavior of flowcondensation on superhydrophobic surfaces.

Adiabatic flows composed of a liquid and gas are commonly studied to gain insight into condensing flows. In adiabatic flows, the vapor fraction and flow regime do not change along the length of the channel, thereby isolating study of the hydrodynamic phenomena (i.e. pressure drop and flow regime behavior). In order to lay the foundation for future research with condensing flows, a flow set-up was constructed to investigate an adiabatic mixture of deionized water and air on superhydrophobic surfaces.

Much research has been done on the pressure drop occurring in two-phase flows. Early work by Lockhart and Martinelli [7] and Chisholm and Laird [8] is often cited as a basis for other work in the literature. Martinelli et al. define two non-dimensional parameters to characterize two-phase flows. First, a nondimensional pressure gradient, $\phi$, is defined as the two-phase flow pressure gradient divided by the pressure gradient that would occur if liquid were the only fluid in the channel, as shown in 


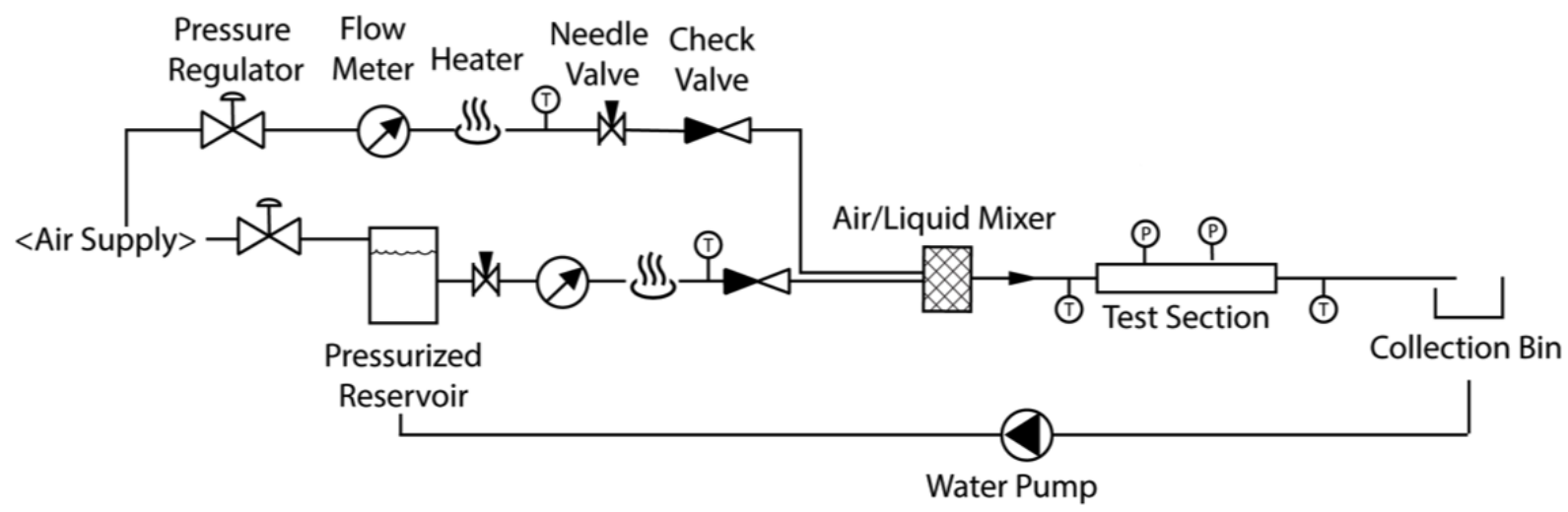

Figure 2. Adiabatic Flow Loop

Equation 1. The dimensionless Martinelli parameter, $\chi$, is a ratio of the pressure gradients for each phase (pressure gradient of the liquid phase only over the pressure gradient for the gas phase only), as shown in Equation 2.

$$
\begin{array}{ll}
\phi_{L}^{2}=\left(\frac{d p}{d z} F\right)_{T P} /\left(\frac{d p}{d z} F\right)_{L} & \text { Eq. } 1 \\
\chi=\left[\left(\frac{d p}{d z} F\right)_{L} /\left(\frac{d p}{d z} F\right)_{G}\right]^{1 / 2} & \text { Eq. } 2
\end{array}
$$

Since their work, dozens of papers have been published with corrections to the original predictions for different fluids, flow rates, and channel shapes and sizes. Several good reviews are provided by Sun and Mishima [9], Asadi et al. [10], and Kim and Mudawar [11] for mini- and microchannel flow. Kim and Mudawar published a universal correlation for a wide range of fluids, flow rates, and channel shapes, constructed from over 7000 data points compiled from 36 studies. The present work compares the pressure drop measurements taken in a classical channel with the predictions from Kim as a preliminary benchmarking step before examining the effect of superhydrophobic channels on pressure drop.

\section{Methods}

A two-phase flow loop was constructed, as shown in Figure 2. The channel cross-section is $1 \mathrm{~mm}$ by $10 \mathrm{~mm}$ and made of acrylic to allow visual access (in order to study flow regime behavior in future studies). The channel is $45 \mathrm{~cm}$ long, and pressure taps are located 15 centimeters from the entrance and exit in order to eliminate entrance and exit effects. House air enters and passes through a gas flow meter (Omega, FLR1004-D) and in-line resistance heater, manually controlled by a variable resistor. Deionized water, pressurized by house air to avoid fluctuations from a pump, enters a liquid flow meter (Omega, FLR1009-D) and an in-line heater (Infinity Fluids, CRES-ILB-24-0040-K-PTC) with a solid state controller. Both fluids are heated in order to more closely match fluid properties during condensation. The air and water are brought together in a 1/16 inch Tjunction, which mixes the phases, before entering a rectangular test section. Two check valves are located upstream of the T-junction in order to prevent back-flow of one phase into the other line. The pressure is measured with a differential pressure transducer (Omega, PX409$2.5 \mathrm{DWU} 5 \mathrm{~V}$ ), and the temperature of the fluids is measured after leaving the heaters, before entering, and after leaving the test section with T-type thermocouples. A data acquisition system ( $\mathrm{NI}, 9205$ and 9214) reads the data at $300 \mathrm{~Hz}$ into LabView where it is filtered (Butterworth low pass filter, $45 \mathrm{~Hz}$ ) and recorded. The flow is allowed to run for a minimum of 60 seconds to reach quasi-steady behavior. Due of the large fluctuations in the pressure signal due to the two phases passing the pressure taps, the pressure signal is averaged over several seconds. 


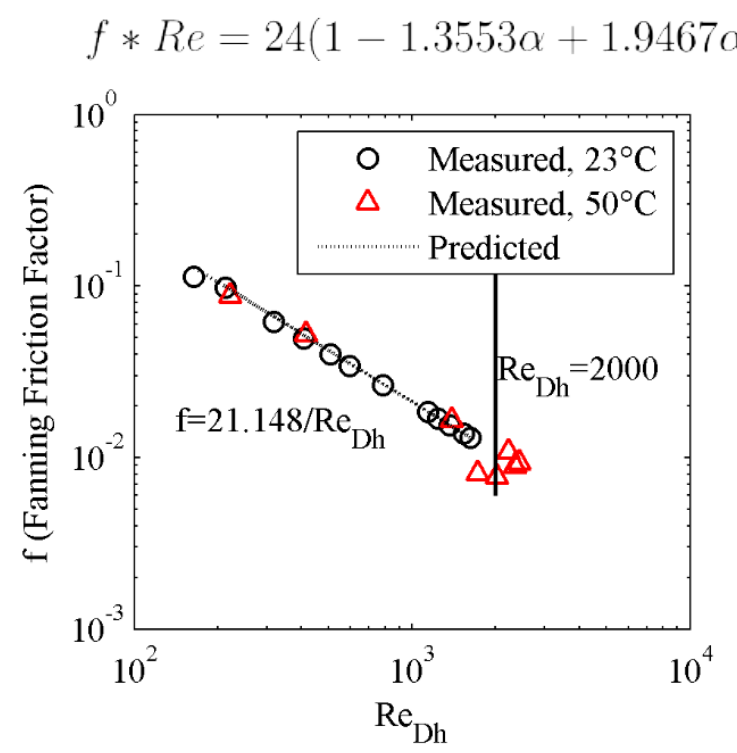

Figure 3. Single-phase pressure drop measurements, predicted from Shah and London [5].

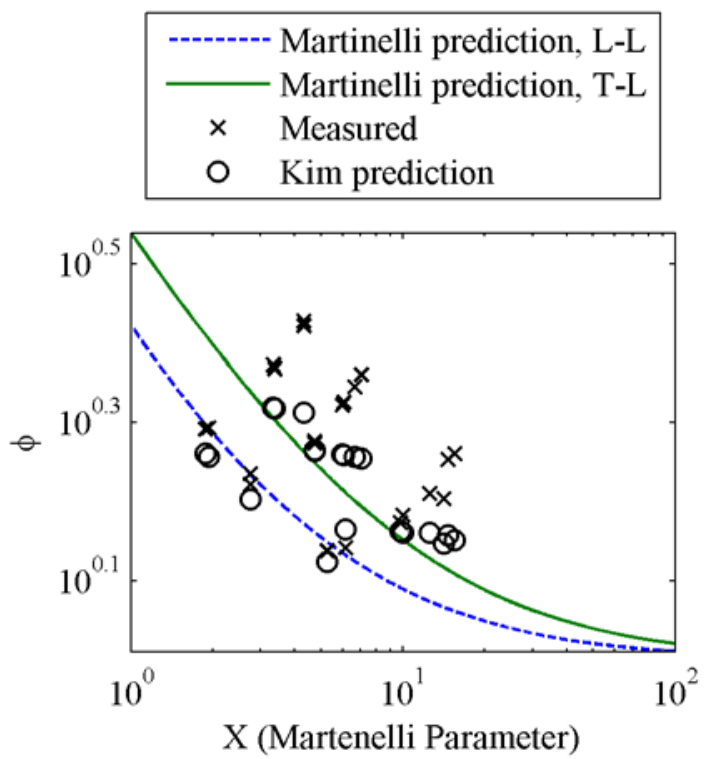

Figure 5: Measured two-phase pressure compared with the predictions of Chisholm [8] and Kim. [11] The designation $L-L$ indicates both the gas and liquid phases are laminar, while T-L indicates turbulent liquid and laminar gas phase.

\section{Results}

As a validation for the setup, pressure drop for single-phase (deionized water) flow in a channel with a classical surface was measured and is presented in Figure 3. The vertical solid line indicates a Reynolds number of 2000 and the onset of turbulence where laminar pressure drop

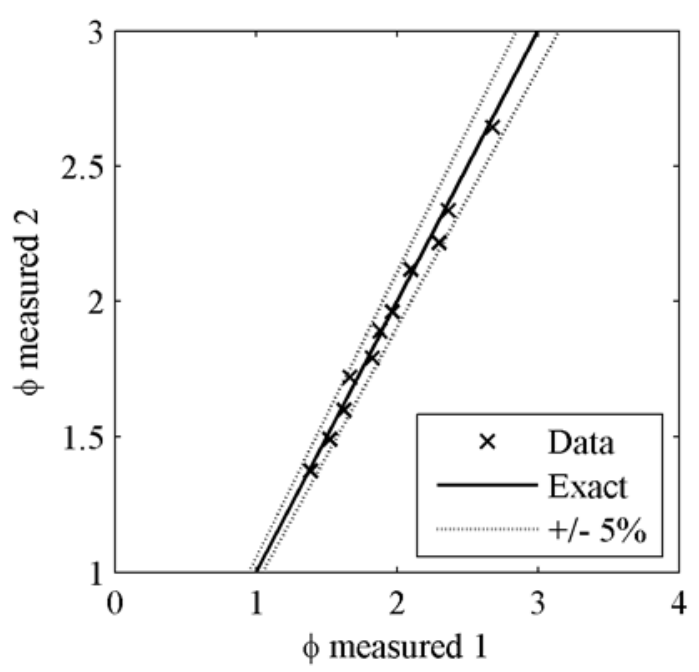

Figure 4: Two-Phase pressure drop measurements from two different days of taking data, showing repeatability

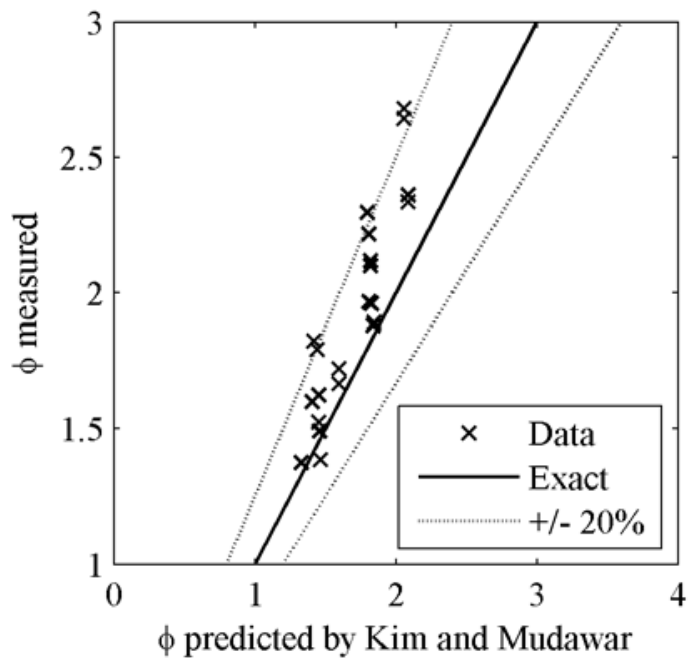

Figure 6. Two-phase pressure drop measurements compared with Kim and Mudawar [11].

correlations are no longer valid. The predicted drop is given in Equation 3 as a function of the channel aspect ratio, $\alpha$ (width divided by height) [12]. Within the laminar region there is excellent agreement between the single phase pressure measurement and the single-phase predictive model. 
Two-phase pressure drop measurements in channel with classical surfaces were obtained. Liquid flow rates from 50 to 500 $\mathrm{mL} / \mathrm{min}$ and air flow rates from 100 to 1000 $\mathrm{mL} / \mathrm{min}$ were measured, corresponding to mass fluxes of 125 to $835 \mathrm{~kg} / \mathrm{m}^{2} \mathrm{~s}$ and .2 to $2 \mathrm{~kg} / \mathrm{m}^{2} \mathrm{~s}$ respectively). The measurements are repeatable to within 5\%, as shown in Figure 4. The data was compared with correlations from Kim and Mudawar [11], with good agreement, as shown in Figures 5 and 6 . The database Kim used to develop their correlation is comprised of data from numerous researchers and experimental conditions (fluid type, channel shapes and sizes, flow rates). As a result, the agreement between the correlation and the database is about $30 \%$. As shown in Figure 3, the measured data matches the correlation by Kim and Mudawar to within $\sim 20 \%$, giving confidence in the accuracy of the two-phase pressure drop measurements.

\section{Conclusions}

The single and two-phase flow pressure drops for a classical channel agree well with correlations found in the literature for a rectangular channel. With this background, the impact of superhydrophobic surfaces on twophase flow can now be explored. The authors plan to characterize and compare pressure drop in channels with a hydrophobic, superhydrophobic, and structured hydrophilic surface. Flow regime behavior in classical and superhydrophobic channels will be explored. The effect of temperature and surface structure (e.g., posts or ribs, cavity fraction) on pressure drop and flow regime will be investigated. The understanding gained from adiabatic two-phase flow work will provide insight that can be applied to condensing flows in superhydrophobic channels.

The use of superhydrophobic surfaces in condensers has the potential to be transformative in not only the power industry, but any process in which condensation occurs. Specifically, loop heat pipes use evaporation and condensation to transfer heat in space environments and have become a popular thermal management component in satellites and other NASA spacecraft [13]. The proposed research has the potential to improve the effectiveness of loop heat pipes. Further, the need for power is ubiquitous in the present generation and is unlikely to diminish in the future. Steam-Rankine cycles provide much of the power utilized in the world today, from a wide variety of energy sources. Improvement in the condenser efficiency may enable the protection of precious water resources through dry-cooling. The present research leads the way for greater understanding of condensation in superhydrophobic channels.

\section{References}

1. Rose, J.W., Dropwise condensation theory and experiment: a review. Proceedings of the Institution of Mechanical Engineers Part a-Journal of Power and Energy, 2002. 216(A2): p. 115-128.

2. Miljkovic, N., et al., Jumping-dropletenhanced condensation on scalable superhydrophobic nanostructured surfaces. Nano Letters, 2013. 13(1): p. 179-87.

3. Miljkovic, N., R. Enright, and E.N. Wang, Effect of droplet morphology on growth dynamics and heat transfer during condensation on superhydrophobic nanostructured surfaces. ACS Nano, 2012. 6(2): p. 1776-1785.

4. Miljkovic, N. and E.N. Wang, Condensation heat transfer on superhydrophobic surfaces. MRS Bulletin, 2013. 38: p. 397-406.

5. Rykaczewski, K., Microdroplet growth mechanism during water condensation on superhydrophobic surfaces. Langmuir, 2012. 28(20): p. 7720-7729.

6. Torresin, D., et al., Flow condensation on copper-based nanotextured superhydrophobic surfaces. Langmuir, 2013. 29(2): p. 840-848.

7. Lockhart, R. and R. Martinelli, Proposed correlation of data for isothermal twophase, two-component flow in pipes. Chem. Eng. Prog, 1949. 45(1): p. 39-48. 
8. Chisholm, D. and A. Laird, Two-phase flow in rough tubes. Trans. ASME, 1958. 80(2): p. 276-286.

9. Sun, L. and K. Mishima, Evaluation analysis of prediction methods for twophase flow pressure drop in minichannels. International Journal of Multiphase Flow, 2009. 35(1): p. 47-54.

10. Asadi, M., G. Xie, and B. Sunden, $A$ review of heat transfer and pressure drop characteristics of single and twophase microchannels. International Journal of Heat and Mass Transfer, 2014. 79(0): p. 34-53.

11. Kim, S.M. and I. Mudawar, Universal approach to predicting two-phase frictional pressure drop for adiabatic and condensing mini/micro-channel flows. International Journal of Heat and Mass Transfer, 2012. 55(11-12): p. 3246-3261.

12. Shah, R.K. and A.L. London, Laminar Flow Forced Convection in Ducts, in Advances in Heat Transfer, J. Thomas F. Irvine and J.P. Hartnett, Editors. 1978, Academic Press.

13. Ku, J., Operating Characteristics of Loop Heat Pipes in 29th International Conference on Environmental System. 1999: Denver, Colorado. 